\title{
Random Pattern Testability Enhancement by Circuit Rewiring*
}

\author{
SHIH-CHIEH CHANG ${ }^{\mathrm{a}}, \mathrm{KWEN}-Y$ O CHEN ${ }^{\mathrm{a}}$, CHING-HWA CHENG ${ }^{\mathrm{a}}$, \\ WEN-BEN JONE ${ }^{c}$ and SUNIL R. DAS ${ }^{\mathrm{b}, \dagger}$

\begin{abstract}
${ }^{a}$ Department of Computer Science and Information Engineering, National Chung Cheng University, Chiayi, Taiwan 621, Republic of China; ${ }^{\mathrm{b}}$ School of Information Technology and Engineering, University of Ottawa,
\end{abstract} \\ Ottawa, Ontario K1N 6N5, Canada; ${ }^{\circ}$ Department of Electrical and Computer Engineering and Computer Science, \\ University of Cincinnati, Cincinnati, OH 45221, USA
}

(Received 15 August 1999; In final form 11 September 2000)

\begin{abstract}
Generally, there exist random-pattern resistant faults that result in the poor fault coverage in Build-In Self-Testing (BIST) scheme. In this paper, we propose a method to enhance the random pattern testability by a circuit restructuring technique, called circuit rewiring. The basic idea of rewiring is to replace a wire by another wire with the circuit functionality remaining unchanged. For two types of rewiring, fanin rewiring and fanout rewiring, we first analyze the testability change for each type of wire replacement. Based on the analysis, an efficient algorithm is given to enhance circuit testability. For a poor observability node, we try to increase its observability by adding an additional fanout to the node and removing an alternative wire whose source node has relatively good observability. The technique does not introduce any hardware overhead and performance degradation since a wire addition is followed immediately by another wire removal. Thus, it is basically cost-free when compared to other testability enhancement techniques.
\end{abstract}

Keywords: Circuit rewiring; Alternative wire; Dominator; Testability; Observability; Random pattern

\section{INTRODUCTION}

Testing a digital circuit is an experiment in which the circuit under test (CUT) is exercised by applying a sequence of input test patterns and then its output responses are analyzed to ascertain whether there exist faults in the circuit. Built-In
Self-Testing (BIST) has been widely used in testing modern circuits or chips, and the basic idea is to have the circuit test itself $[1,2]$. Basically, BIST of logic circuits can be divided into two major categories: random testing and exhaustive testing. By embedding a built-in device such as linear feedback shift register (LFSR) inside the chip, we

\footnotetext{
* This research was supported in part by the Natural Sciences and Engineering Research Council of Canada under Grant A 4750.

${ }^{\dagger}$ Corresponding author.
} 
can have a sequence of random test patterns generated if the LFSR is initiated with a proper seed value. The defects that occur can be sensitized and detected if the number of random test patterns is large. Of course, there is no real random pattern generator as long as the machine is built by human, since bit dependency occurs. However, the test vectors generated still have many properties of random patterns and are called pseudo-random test patterns [3]. The problem with pseudo-random testing lies in the difficulty of enhancing the random pattern testability for circuits containing many random-pattern resistant faults.

Basically, the random-pattern resistance problem is solved by applying weighted random patterns $[4,14,19]$ or by inserting test points [13, 15-18]. The basic idea of weighted random pattern testing is to test the logic with biased random patterns, i.e., the numbers of logic 1 and logic 0 applied to each input are not equal. This can be achieved by building a weighted random pattern generator from an LFSR with AND/OR gates. Substantial improvement can be obtained if the test patterns are properly weighted. Instead of changing the distribution of random patterns, the other major approach is to modify the logic by inserting test points such that the detection probabilities of all random-pattern resistant faults can be increased [15]. Both approaches incur hardware overhead and performance degradation.

In this work, we propose a method that is able to enhance the random pattern testability of the CUT by a circuit restructuring technique called circuit rewiring. The rewiring technique for testability enhancement has the potential of no hardware overhead and performance degradation. The basic idea of circuit rewiring is to find a wire to replace another one without changing the circuit function. Thus, it is beneficial to supersede a hardto-test line using another easy-to-test line. Given a set of random test patterns, all undetected faults can be identified by fault simulation. The fault coverage accomplished by the set of random patterns can be further increased by circuit rewiring. In this experiment, we try to rewire the circuit such that the observabilities of undetected lines can be enhanced. Simulation results demonstrate that the circuit rewiring technique can successfully break the random pattern barrier for many benchmark circuits. Without rewiring, the fault coverage of a CUT might saturate and remain a constant regardless of more test patterns being applied. However, the fault coverage can be made higher if circuit lines are properly rewired. We have observed that circuit rewiring increases random pattern fault coverage in most cases.

The background of circuit rewiring is described in Section 2, while Section 3 investigates the relationship between circuit rewiring and random pattern testability, and a heuristic rewiring method is proposed. Simulation results are provided in Section 4, and Section 5 concludes the work.

\section{BACKGROUND}

This paper applies the idea of rewiring to improve the testability of a circuit. There have been several studies $[5-7,10,12]$ on how to logically exchange wire connections under the constraint that the circuit function remains unchanged. In this section, we will review the basic concept of rewiring which has the ability of modifying a circuit. In the next section, an algorithm using circuit rewiring for testability enhancement will be presented.

By definition, wire $\mathrm{A}$ is an alternative wire of wire B, if we can replace wire B by wire A. Consider the circuit shown in Figure 1, wire $g_{1} \rightarrow g_{4}$ can be replaced by wire $g_{5} \rightarrow g_{9}$ with the circuit function remaining unchanged, i.e., both circuits

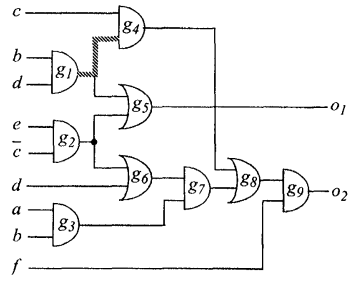

(a)

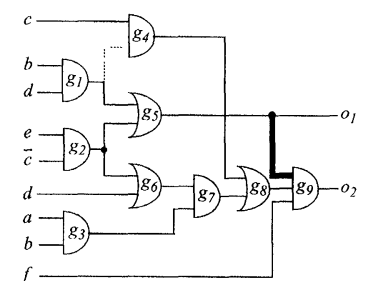

(b)
FIGURE 1 An example for single alternative wire replacement. 
in Figure 1 are functionally equivalent. In this case, we say that wire $g_{5} \rightarrow g_{9}$ is an alternative wire of wire $g_{1} \rightarrow g_{4}$. Additionally, the alternative wires are mutually alternative, i.e., if wire $\mathrm{A}$ is an alternative wire of wire $B$ then wire $B$ is also an alternative wire of wire A. Thus, we can also replace $g_{5} \rightarrow g_{9}$ by $g_{1} \rightarrow g_{4}$ in Figure 1 .

Let us further examine the same example. First, a redundant wire is a wire that can be added (removed) to (from) a circuit without changing the circuit function. It can be found that the nonexisting wire $g_{5} \rightarrow g_{9}$ is redundant in Figure 1(a) and can be added to the circuit. On the other hand, $g_{1} \rightarrow g_{4}$ is ir-redundant in Figure 1(a). However, when wire $g_{5} \rightarrow g_{9}$ is added to the circuit, wire $g_{1} \rightarrow g_{4}$ becomes redundant and can thus be removed from the circuit (Fig. 1(b)). From this example, we can observe that an originally irredundant wire may become redundant after adding some redundant wire to a circuit. The way of redundancy addition and removal forms the basic idea for the circuit rewiring technique.

The procedure of finding an alternative wire for the target wire first searches a non-existing wire, called candidate connection, that once added makes the target wire redundant. Then, the rewiring technique checks whether the candidate connection is redundant, i.e., whether adding the non-existing wire preserves the circuit functionality. It is only when the candidate connection is verified as redundant, we can then add the candidate connection and further remove the target wire. All redundancy checks can be done through reasoning of automatic test pattern generation (ATPG). More details of finding alternative wires can be found in [7].

\section{REWIRING FOR TESTABILITY}

In the section, the effect of rewiring to circuit testability is first analyzed, and then a heuristic method for testability enhancement will be presented. In order to test a stuck-at fault, it is required to activate the fault and then propagate the fault effect to at least one primary output. By definition, the probability of a primary input vector to activate a fault $f$ is called the controllability of $f$, while that to propagate the fault effect of $f$ to a primary output is called the observability of $f$. Both controllability and observability of a fault adequately indicate the degree of difficulty to activate the fault and to observe the fault effect. Thus, the testability of a fault can be measured by these two values.

For observability, in general, if a node is close to a primary output, then the observability of the node is high. A primary output node is considered to be fully observable. Further, if a node has many fanouts, the node has good opportunity to be highly observable because the fault effect can propagate to primary outputs from one of its fanouts. (We neglect the fault-masking problem.) In a circuit, some nodes may have very good observability such as a primary output node or a node with many fanouts while some nodes may have very poor observability. Since each iteration of the rewiring process allows to add/remove a wire at the fanout of a certain node, the basic idea of our approach is to improve the low observability of a node by adding new fanouts to this node. Thus, special types of rewiring extended from [6] are analyzed, and the effects to circuit testability caused by such rewirings are then investigated. Before further discussion, several definitions are provided. Node B is a transitive fanout of node $A$, if there exists a path from node $\mathrm{A}$ to node $\mathrm{B}$. Node $\mathrm{A}$ is a dominator of node $\mathrm{B}$ (wire $\mathrm{C}$ ), if every path from node $\mathrm{B}$ (wire C) to any primary output must pass through node A. For example, in Figure 1(a), nodes $g_{8}$ and $g_{9}$ are dominators of both node $g_{4}$ and wire $g_{1} \rightarrow g_{4}$.

\subsection{Two Special Types of Rewiring}

Here, we will describe two special types of rewiring and the first one is named the fanout rewiring. In this case, a fanout wire $g_{\mathrm{a}} \rightarrow g_{\mathrm{b}}$ of gate $g_{\mathrm{a}}$ dotted in Figure 2(a) is replaced by another fanout wire 
$g_{\mathrm{a}} \rightarrow g_{\mathrm{d}}$ bold in Figure 2(b); additionally, node $g_{\mathrm{d}}$ is restricted to be a dominator of $g_{\mathrm{b}}$ and both gates $g_{\mathrm{d}}$ and $g_{\mathrm{b}}$ must have the same type of function, i.e., they are both OR gates as in the figure or AND gates. In Figure 2, let Cone II contain all the wires and gates that are the transitive fanouts of $g_{\mathrm{a}}$ and are dominated by $g_{\mathrm{d}}$, while let Cone I contain all the wires dominated by $g_{\mathrm{a}}$. We have the following theorem.

TheOREM 1 Suppose wire $g_{a} \rightarrow g_{b}$ can be replaced by $g_{a} \rightarrow g_{d}$ where $g_{d}$ is a dominator of $g_{b}$. A test pattern which an detect a stuck-at fault in Cone II after rewiring (Fig. 2(b)), can also detect the same fault before rewiring (Fig. 2(a)). Further, if a test vector can detect a fault in Cone I before rewiring (Fig. 2(a)), the same vector can also detect the fault after rewiring (Fig. 2(b)).

Proof Consider testing line $w$ stuck-at fault in the rewired circuit shown in Figure 2(b). Because $g_{d}$ is a dominator of $w$, the side-input $g_{\text {a }}$ must be assigned the noncontrolling value 0 . Assigning 0 to $g_{\mathrm{a}}$ will cause both wires $g_{\mathrm{a}} \rightarrow g_{\mathrm{d}}$ in Figure 2(b) and $g_{\mathrm{a}} \rightarrow g_{\mathrm{b}}$ in Figure 2(a) to be "transparent", i.e., their presence will not affect the result. Therefore, any test pattern which can detect $w$ stuck-at fault for the circuit after line substitution is also a test pattern for the same fault in the circuit before line substitution. The case for Cone I and other gate types can be proved similarly.

Q.E.D.

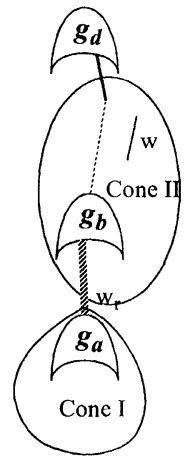

(a)
Cone II testability decrease

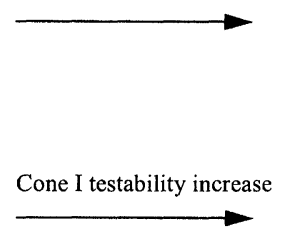

FIGURE 2 Fanout rewiring.
LEMMA 1 If wire $g_{a} \rightarrow g_{b}$ is replaced by $g_{a} \rightarrow g_{d}$, the testability of a fault in Cone II decreases while that in Cone I increases.

Proof This can be directly proved from Theorem 1.

The intuition behind the above theorem and lemma is that after replacing $g_{a} \rightarrow g_{b}$ by $g_{a} \rightarrow g_{d}$, node $g_{\mathrm{a}}$ has a fanout wire which is closer to the primary outputs, as a result, the observability of $g_{a}$ increases and so do the wires dominated by $g_{a}$. On the other hand, the wires in Cone II has the additional constraint, $g_{a}$ must be 0 during testing; therefore, the testability for wires in Cone II will decrease after wire replacement.

Another type of rewiring is referred as the fanin rewiring. Let the fanin wire $g_{\mathrm{a}} \rightarrow g_{\mathrm{d}}$ of gate $g_{\mathrm{d}}$ dotted in Figure 3(a) be replaced by another fanin wire $g_{\mathrm{b}} \rightarrow g_{\mathrm{d}}$ bold in Figure 3(b). Further, wires dominated by $g_{\mathrm{a}}$ are referred as Cone III and wires dominated by $g_{\mathrm{b}}$ are referred as Cone IV. Suppose there is no multiple fault cancellation problem, the observability of the wires in Cone III will decrease after rewiring because one fanout wire of the gate $g_{\mathrm{a}}$ has been deleted. Since an additional fanout wire is added to gate $g_{\mathrm{b}}$, the observability of wires in Cone IV will potentially increase.

The change of testability after rewiring can be used to guide wire replacement to improve the circuit testability. For example, since the primary outputs are fully observable, there is no loss of

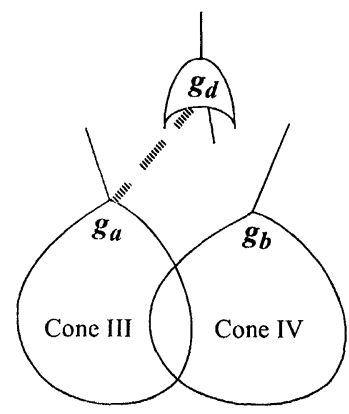

(a)

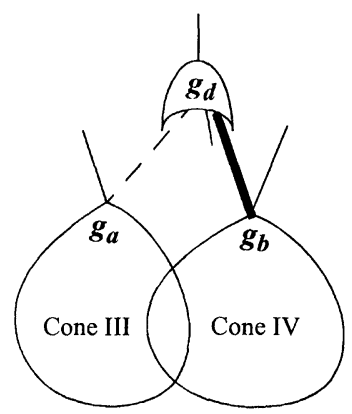

(b)
FIGURE 3 Fanin rewiring. 
observability by removing any of its fanouts. Consider wire $g_{5} \rightarrow g_{9}$ in Figure 1(b), since $g_{5}$ is directly connected to primary output $O_{1}$, node $g_{5}$ is fully observable. Removing another fanout from $g_{5}$ will not decrease the observability of $g_{5}$ at all. Therefore, replacing $g_{5} \rightarrow g_{9}$ by $g_{1} \rightarrow g_{4}$ will not decease the observability of $g_{5}$ but may increase the observability of $g_{1}$ because the number of $g_{1}$ 's fanouts is increased by one.

\subsection{A Heuristic for Improving Testability}

There are two possibilities in using the circuit rewiring technique for testability enhancement. The first one tries to improve the testability of a CUT without considering what kind of patterns are applied, while the second approach tries to improve the fault coverage for a given set of patterns.

In the first approach, we estimate the fault coverage of the CUT. The estimation can be done by applying testability analysis methods $[2,8$, $9,11]$. For each rewiring, the fault coverage of the CUT can be estimated again. In the rewiring technique, when a wire is added to improve the observability of node $a$, the fanout wire of another node $b$ must be removed and the observability of node $b$ will be reduced. In order to improve the observability of a node, we apply the first approach that removes a wire whose source node has more fanouts to one whose source node has less fanouts. In this way, we are able to "shift" some testability from high testability nodes to low testability nodes.

In the second approach, we assume that a set of random or deterministic test patterns has been given and try to improve the fault coverage for these patterns. First, the patterns are simulated and faults that are not covered by these patterns are identified. Then, we index nodes in such a way that a node that is the transitive fanouts of many undetected faults is given a large number. The objective in this approach is to improve the observabilities for nodes with large indices because increasing the observabilities for these nodes can greatly improve the observabilities of many undetected faults. We will use the same technique as the one used in the first approach to add new fanouts for nodes with large indices. After each rewiring, the circuit must be re-simulated because the fault detection behavior might be changed after each iteration of rewiring step has been taken. That is, the rewiring process can not guarantee the detection of undetected faults; even worse, some detected faults might turn to be undetected. Thus, fault simulation must be performed for each iteration to guarantee that the rewiring process is beneficial.

In the third approach, we estimate the observability of each node in the CUT. Similar to the second approch, the objective of this approach is to improve the observabilities of the nodes with lower observabilities, and also uses the same technique as the one used in the first approach to add new fanouts for nodes with lower observabilities. After each rewiring, we also re-simulate the circuit to estimate the observability of each node in the CUT again.

Consider the example shown in Figure 4(a) with test vector $(a, b, c, d, e)=(0,1,0,1,0)$. After fault simulation using the test vector, it can be found that stem line $g_{1}$ stuck-at 1 cannot be detected, and

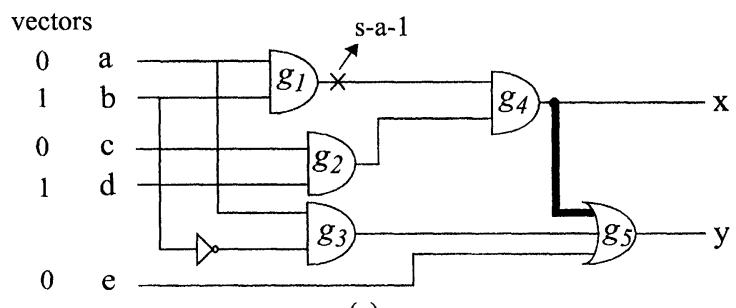

(a)

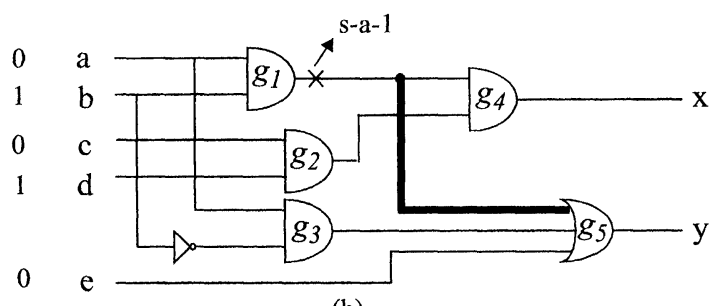

(b)

FIGURE 4 An example for testability enhancement. 
TABLE I Simulation results (approach 1)

\begin{tabular}{|c|c|c|c|c|c|c|c|}
\hline Circuit & $\begin{array}{l}\text { \# of } \\
\text { faults }\end{array}$ & $\begin{array}{c}\text { \# of random } \\
\text { patterns }\end{array}$ & $\begin{array}{l}\text { \# of DF } \\
\text { of ckt.org }\end{array}$ & $\begin{array}{l}\mathrm{FC} \text { of } \\
\text { ckt.org }\end{array}$ & $\begin{array}{l}\text { \# of DF } \\
\text { of ckt.rm }\end{array}$ & FC of ckt.rm & $\begin{array}{l}\text { Time } \\
(\mathrm{sec})\end{array}$ \\
\hline 9symml & 774 & $\begin{array}{r}500 \\
1000\end{array}$ & $\begin{array}{l}709 \\
760\end{array}$ & $\begin{array}{l}91.60 \% \\
98.19 \%\end{array}$ & $\begin{array}{l}740 \\
768\end{array}$ & $\begin{array}{l}95.61 \\
99.22\end{array}$ & $\begin{array}{l}393.25 \\
488.11\end{array}$ \\
\hline $\mathrm{C} 1355$ & 1984 & 500 & 1876 & $94.56 \%$ & 1876 & 94.56 & \\
\hline C1908 & 2116 & 500 & 1804 & $85.26 \%$ & 1804 & 85.26 & 7418.50 \\
\hline $\mathrm{C} 2670$ & 3090 & 1000 & 2498 & $81.68 \%$ & 2561 & 82.88 & 26660.14 \\
\hline C3540 & 4440 & $\begin{array}{l}5000 \\
2000\end{array}$ & $\begin{array}{l}2537 \\
4222\end{array}$ & $\begin{array}{l}82.10 \% \\
95.09 \%\end{array}$ & $\begin{array}{l}2572 \\
4222\end{array}$ & $\begin{array}{l}83.24 \\
95.09\end{array}$ & $\begin{array}{l}48510.96 \\
81503.50\end{array}$ \\
\hline \multirow[t]{2}{*}{$\mathrm{C} 432$} & 736 & 500 & 690 & $93.75 \%$ & 692 & 94.02 & 189.08 \\
\hline & & $\begin{array}{r}1000 \\
* 50000\end{array}$ & $\begin{array}{l}690 \\
690\end{array}$ & $\begin{array}{l}93.75 \% \\
93.75 \%\end{array}$ & $\begin{array}{l}692 \\
692\end{array}$ & $\begin{array}{l}94.02 \\
94.02\end{array}$ & $\begin{array}{r}234.10 \\
2495.35\end{array}$ \\
\hline \multirow[t]{5}{*}{ C499 } & 1568 & 500 & 1494 & $95.28 \%$ & 1502 & 95.79 & 657.18 \\
\hline & & 1000 & 1535 & $97.90 \%$ & 1543 & 98.41 & 721.06 \\
\hline & & 2000 & 1558 & $99.36 \%$ & 1566 & 99.87 & 894.10 \\
\hline & & 5000 & 1560 & $99.49 \%$ & 1568 & 100.00 & 1366.11 \\
\hline & & ${ }^{*} 100000$ & 1560 & $99.49 \%$ & 1568 & 100.00 & 19641.02 \\
\hline C6288 & 9408 & 500 & 9404 & $99.96 \%$ & 9404 & 99.96 & 20599.70 \\
\hline \multirow[t]{3}{*}{$\mathrm{C} 880$} & 1280 & 1000 & 1221 & $95.39 \%$ & 1225 & 95.70 & 1011.71 \\
\hline & & 5000 & 1259 & $98.36 \%$ & 1260 & 98.44 & 1980.14 \\
\hline & & 10000 & 1268 & $99.06 \%$ & 1268 & 99.06 & 3040.29 \\
\hline \multirow[t]{3}{*}{ alu2 } & 1762 & 1000 & 1464 & $83.09 \%$ & 1532 & 86.95 & 8561.32 \\
\hline & & 3000 & 1481 & $84.05 \%$ & 1547 & 87.80 & 12216.92 \\
\hline & & $* 50000$ & 1481 & $84.05 \%$ & 1547 & 87.80 & 108144.58 \\
\hline alu4 & 3164 & 5000 & 2775 & $87.71 \%$ & 2830 & 89.44 & 44578.30 \\
\hline \multirow[t]{2}{*}{ apex6 } & 2432 & 5000 & 2356 & $96.88 \%$ & 2374 & 97.62 & 14933.60 \\
\hline & & 10000 & 2383 & $97.99 \%$ & 2394 & 98.44 & 23964.05 \\
\hline \multirow[t]{6}{*}{ apex7 } & 922 & 500 & 831 & $90.13 \%$ & 842 & 91.32 & 1152.18 \\
\hline & & 1000 & 837 & $90.78 \%$ & 848 & 91.97 & 685.06 \\
\hline & & 5000 & 849 & $92.08 \%$ & 860 & 93.28 & 1314.10 \\
\hline & & 10000 & 859 & $93.17 \%$ & 869 & 94.25 & 2180.25 \\
\hline & & 20000 & 863 & $93.60 \%$ & 872 & 94.58 & 3886.33 \\
\hline & & ${ }^{*} 100000$ & 863 & $93.60 \%$ & 872 & 94.58 & 19149.90 \\
\hline \multirow[t]{2}{*}{ b1 } & 48 & 500 & 30 & $62.50 \%$ & 34 & 70.83 & 0.01 \\
\hline & & ${ }^{*} 100000$ & 30 & $62.50 \%$ & 34 & 70.83 & 4.01 \\
\hline \multirow[t]{3}{*}{ b9 } & 480 & 500 & 465 & $96.88 \%$ & 472 & 98.33 & 120.06 \\
\hline & & 1000 & 471 & $98.12 \%$ & 476 & 99.17 & 140.06 \\
\hline & & $* 50000$ & 471 & $98.12 \%$ & 476 & 99.17 & 1790.56 \\
\hline \multirow[t]{2}{*}{ c8 } & 930 & 1000 & 758 & $81.51 \%$ & 762 & 81.94 & 439.07 \\
\hline & & 5000 & 787 & $84.62 \%$ & 787 & 84.62 & 907.10 \\
\hline \multirow[t]{2}{*}{$\mathrm{cc}$} & 262 & 100 & 228 & $87.02 \%$ & 236 & 90.08 & 11.02 \\
\hline & & 200 & 258 & $98.47 \%$ & 259 & 98.85 & 14.02 \\
\hline \multirow[t]{2}{*}{$\mathrm{cm} 85 \mathrm{a}$} & 176 & 100 & 156 & $88.64 \%$ & 163 & 92.61 & 4.02 \\
\hline & & 200 & 174 & $98.86 \%$ & 175 & 99.43 & 4.02 \\
\hline \multirow[t]{3}{*}{$\mathrm{cmb}$} & 164 & 1000 & 103 & $62.80 \%$ & 106 & 64.63 & 3.02 \\
\hline & & 5000 & 138 & $84.15 \%$ & 141 & 85.98 & 7.01 \\
\hline & & 10000 & 150 & $91.46 \%$ & 150 & 91.46 & 12.02 \\
\hline \multirow[t]{3}{*}{ comp } & 510 & 5000 & 304 & $59.61 \%$ & 325 & 63.73 & 169.12 \\
\hline & & 10000 & 326 & $63.92 \%$ & 343 & 67.25 & 248.15 \\
\hline & & 50000 & 422 & $82.75 \%$ & 437 & 85.69 & 978.67 \\
\hline cordic & 328 & 500 & 256 & $78.05 \%$ & 256 & 78.05 & 43.03 \\
\hline count & 476 & 500 & 387 & $81.30 \%$ & 397 & 83.40 & 43.02 \\
\hline \multirow[t]{4}{*}{$\mathrm{cu}$} & 230 & 500 & 204 & $88.70 \%$ & 214 & 93.04 & 17.02 \\
\hline & & 1000 & 205 & $89.13 \%$ & 215 & 93.48 & 19.02 \\
\hline & & 5000 & 211 & $91.74 \%$ & 221 & 96.09 & 39.03 \\
\hline & & $* 50000$ & 212 & $92.17 \%$ & 221 & 96.09 & 64.03 \\
\hline
\end{tabular}


TABLE II Simulation results (approach 2)

\begin{tabular}{|c|c|c|c|c|c|c|c|}
\hline Circuit & $\begin{array}{l}\text { \# of } \\
\text { faults }\end{array}$ & $\begin{array}{c}\text { \# of random } \\
\text { patterns }\end{array}$ & $\begin{array}{l}\text { \# of DF } \\
\text { of ckt.org }\end{array}$ & $\begin{array}{l}\text { FC of } \\
\text { ckt.org }\end{array}$ & $\begin{array}{l}\text { \# of DF } \\
\text { of ckt.rm }\end{array}$ & FC of ckt.rm & $\begin{array}{l}\text { Time } \\
(\mathrm{sec})\end{array}$ \\
\hline \multirow[t]{3}{*}{ 9symml } & 774 & 500 & 709 & 91.60 & 755 & $97.55 \%$ & 556.68 \\
\hline & & 1000 & 760 & 98.19 & 773 & $99.87 \%$ & 435.56 \\
\hline & & 2000 & 771 & 99.61 & 774 & $100.00 \%$ & 233.07 \\
\hline $\mathrm{C} 1355$ & 1984 & 500 & 1876 & 94.56 & 1876 & $94.56 \%$ & 54.07 \\
\hline C1908 & 2116 & 500 & 1804 & 85.26 & 1804 & $85.26 \%$ & 6487.14 \\
\hline \multirow[t]{2}{*}{$\mathrm{C} 2670$} & 3090 & 1000 & 2498 & 81.68 & 2550 & $82.52 \%$ & 11826.18 \\
\hline & & 5000 & 2537 & 82.10 & 2555 & $82.69 \%$ & 21795.32 \\
\hline C 3540 & 4440 & 2000 & 4222 & 95.09 & 4222 & $95.09 \%$ & 18271.36 \\
\hline \multirow[t]{3}{*}{$\mathrm{C} 432$} & 736 & 500 & 690 & 93.75 & 692 & $94.02 \%$ & 245.41 \\
\hline & & 1000 & 690 & 93.75 & 692 & $94.02 \%$ & 266.08 \\
\hline & & *50000 & 690 & 93.75 & 692 & $94.02 \%$ & 1852.26 \\
\hline \multirow[t]{5}{*}{ C499 } & 1568 & 500 & 1494 & 95.28 & 1502 & $95.79 \%$ & 666.08 \\
\hline & & 1000 & 1535 & 97.90 & 1543 & $98.41 \%$ & 735.17 \\
\hline & & 2000 & 1558 & 99.36 & 1566 & $99.87 \%$ & 904.12 \\
\hline & & 5000 & 1560 & 99.49 & 1568 & $100.00 \%$ & 1403.15 \\
\hline & & ${ }^{*} 100000$ & 1560 & 99.49 & 1568 & $100.00 \%$ & 18953.08 \\
\hline C6288 & 9408 & 500 & 9404 & 99.96 & 9404 & $99.96 \%$ & 1262.23 \\
\hline \multirow[t]{3}{*}{$\mathrm{C} 880$} & 1280 & 1000 & 1221 & 95.39 & 1225 & $95.70 \%$ & 723.66 \\
\hline & & 5000 & 1259 & 98.36 & 1260 & $98.44 \%$ & 451.40 \\
\hline & & 10000 & 1268 & 99.06 & 1268 & $99.06 \%$ & 71.10 \\
\hline \multirow[t]{3}{*}{ alu2 } & 1762 & 1000 & 1464 & 83.09 & 1519 & $86.21 \%$ & 3766.57 \\
\hline & & 3000 & 1481 & 84.05 & 1534 & $87.06 \%$ & 5351.31 \\
\hline & & $* 50000$ & 1481 & 84.05 & 1534 & $87.06 \%$ & 83394.63 \\
\hline alu4 & 3164 & 5000 & 2775 & 87.71 & 2843 & $89.85 \%$ & 53492.16 \\
\hline \multirow[t]{4}{*}{ apex6 } & 2432 & 5000 & 2356 & 96.88 & 2372 & $97.53 \%$ & 3156.88 \\
\hline & & 10000 & 2383 & 97.99 & 2398 & $98.60 \%$ & 3239.53 \\
\hline & & 50000 & 2418 & 99.42 & 2422 & $99.59 \%$ & 9261.08 \\
\hline & & 100000 & 2424 & 99.67 & 2424 & $99.67 \%$ & 7873.49 \\
\hline \multirow[t]{6}{*}{ apex7 } & 922 & 500 & 831 & 90.13 & 840 & $91.11 \%$ & 314.05 \\
\hline & & 1000 & 837 & 90.78 & 845 & $91.65 \%$ & 363.05 \\
\hline & & 5000 & 849 & 92.08 & 857 & $92.95 \%$ & 706.08 \\
\hline & & 10000 & 859 & 93.17 & 866 & $93.93 \%$ & 933.03 \\
\hline & & 20000 & 863 & 93.60 & 870 & $94.36 \%$ & 1692.35 \\
\hline & & ${ }^{*} 100000$ & 863 & 93.60 & 870 & $94.36 \%$ & 8300.22 \\
\hline \multirow[t]{2}{*}{ b1 } & 48 & 500 & 30 & 62.50 & 34 & $70.83 \%$ & 0.01 \\
\hline & & $* 100000$ & 30 & 62.50 & 34 & $70.83 \%$ & 3.02 \\
\hline \multirow[t]{3}{*}{ b9 } & 480 & 500 & 465 & 96.88 & 472 & $98.33 \%$ & 56.10 \\
\hline & & 1000 & 471 & 98.12 & 474 & $98.75 \%$ & 61.09 \\
\hline & & *50000 & 471 & 98.12 & 474 & $98.75 \%$ & 442.12 \\
\hline \multirow[t]{2}{*}{$\mathrm{c} 8$} & 930 & 1000 & 758 & 81.51 & 763 & $82.04 \%$ & 634.91 \\
\hline & & 5000 & 787 & 84.62 & 787 & $84.62 \%$ & 657.02 \\
\hline \multirow[t]{3}{*}{$\mathrm{cc}$} & 262 & 100 & 228 & 87.02 & 233 & $88.93 \%$ & 11.03 \\
\hline & & 200 & 258 & 98.47 & 259 & $98.85 \%$ & 2.01 \\
\hline & & 300 & 262 & 100.00 & 262 & $100.00 \%$ & 0.00 \\
\hline \multirow[t]{3}{*}{$\mathrm{cm} 85 \mathrm{a}$} & 176 & 100 & 156 & 88.64 & 163 & $92.61 \%$ & 4.02 \\
\hline & & 200 & 174 & 98.86 & 175 & $99.43 \%$ & 1.01 \\
\hline & & 300 & 176 & 100.00 & 176 & $100.00 \%$ & 0.00 \\
\hline \multirow[t]{3}{*}{$\mathrm{cmb}$} & 164 & 1000 & 103 & 62.80 & 105 & $64.02 \%$ & 4.02 \\
\hline & & 5000 & 138 & 84.15 & 138 & $84.15 \%$ & 9.02 \\
\hline & & 10000 & 150 & 91.46 & 150 & $91.46 \%$ & 13.03 \\
\hline comp & 510 & 5000 & 304 & 59.61 & 310 & $60.78 \%$ & 38.06 \\
\hline & & 10000 & 326 & 63.92 & 332 & $65.10 \%$ & 69.09 \\
\hline & & 50000 & 422 & 82.75 & 424 & $83.14 \%$ & 289.52 \\
\hline & & 100000 & 435 & 85.29 & 437 & $85.69 \%$ & 311.07 \\
\hline & & 200000 & 445 & 87.25 & 447 & $87.65 \%$ & 637.15 \\
\hline cordic & 328 & 500 & 256 & 78.05 & 257 & $78.35 \%$ & 81.20 \\
\hline $\mathrm{cu}$ & 230 & 500 & 204 & 88.70 & 209 & $90.87 \%$ & 19.06 \\
\hline & & 1000 & 205 & 89.13 & 210 & $91.30 \%$ & 21.05 \\
\hline & & 5000 & 211 & 91.74 & 216 & $93.91 \%$ & 42.09 \\
\hline & & 10000 & 212 & 92.17 & 217 & $94.35 \%$ & 37.02 \\
\hline & & *50000 & 212 & 92.17 & 217 & $94.35 \%$ & 162.08 \\
\hline
\end{tabular}


TABLE III Simulation results (approach 3)

\begin{tabular}{|c|c|c|c|c|c|c|c|}
\hline Circuit & $\begin{array}{l}\# \text { of } \\
\text { faults }\end{array}$ & $\begin{array}{l}\text { \# of random } \\
\text { patterns }\end{array}$ & $\begin{array}{c}\text { \# of DF } \\
\text { of ckt.org }\end{array}$ & $\begin{array}{l}\mathrm{FC} \text { of } \\
\text { ckt.org }\end{array}$ & $\begin{array}{l}\text { \# of DF } \\
\text { of ckt.rm }\end{array}$ & FC of ckt.rm & $\begin{array}{r}\text { Time } \\
(\mathrm{sec})\end{array}$ \\
\hline \multirow[t]{2}{*}{ 9symml } & 774 & 500 & 709 & $91.60 \%$ & 739 & $95.48 \%$ & 371.09 \\
\hline & & 1000 & 760 & $98.19 \%$ & 771 & $99.61 \%$ & 472.10 \\
\hline $\mathrm{C} 1355$ & 1984 & 500 & 1876 & $94.56 \%$ & 1876 & $94.56 \%$ & 127.07 \\
\hline C1908 & 2116 & 500 & 1804 & $85.26 \%$ & 1804 & $85.26 \%$ & 1970.48 \\
\hline \multirow[t]{2}{*}{$\mathrm{C} 2670$} & 3090 & 1000 & 2498 & $81.68 \%$ & 2550 & $82.52 \%$ & 36266.61 \\
\hline & & 5000 & 2537 & $82.10 \%$ & 2563 & $82.94 \%$ & 94808.92 \\
\hline \multirow[t]{2}{*}{ C3540 } & 4440 & 2000 & 4222 & $95.09 \%$ & 4222 & $95.09 \%$ & 49027.77 \\
\hline & & & 4244 & $95.59 \%$ & 4244 & $95.59 \%$ & 72015.88 \\
\hline \multirow[t]{3}{*}{ C432 } & 736 & 500 & 690 & $93.75 \%$ & 692 & $94.02 \%$ & 149.05 \\
\hline & & 1000 & 690 & $93.75 \%$ & 692 & $94.02 \%$ & 169.04 \\
\hline & & $* 50000$ & 690 & $93.75 \%$ & 692 & $94.02 \%$ & 2024.41 \\
\hline \multirow[t]{5}{*}{ C499 } & 1568 & 500 & 1494 & $95.28 \%$ & 1502 & $95.79 \%$ & 632.08 \\
\hline & & 1000 & 1535 & $97.90 \%$ & 1543 & $98.41 \%$ & 744.08 \\
\hline & & 2000 & 1558 & $99.36 \%$ & 1566 & $99.87 \%$ & 881.11 \\
\hline & & 5000 & 1560 & $99.49 \%$ & 1568 & $100.00 \%$ & 1320.17 \\
\hline & & $* 100000$ & 1560 & $99.49 \%$ & 1568 & $100.00 \%$ & 35941.18 \\
\hline C6288 & 9408 & 500 & 9404 & $99.96 \%$ & 9404 & $99.96 \%$ & 32278.51 \\
\hline \multirow[t]{3}{*}{ C880 } & 1280 & 1000 & 1221 & $95.39 \%$ & 1226 & $95.78 \%$ & 1512.29 \\
\hline & & 5000 & 1259 & $98.36 \%$ & 1260 & $98.44 \%$ & 2911.74 \\
\hline & & 10000 & 1268 & $99.06 \%$ & 1268 & $99.06 \%$ & 4003.97 \\
\hline \multirow[t]{3}{*}{ alu2 } & 1762 & 1000 & 1464 & $83.09 \%$ & 1518 & $86.15 \%$ & 3901.32 \\
\hline & & 3000 & 1481 & $84.05 \%$ & 1535 & $87.12 \%$ & 5949.40 \\
\hline & & $* 50000$ & 1481 & $84.05 \%$ & 1535 & $87.12 \%$ & 52435.91 \\
\hline alu4 & 3164 & 5000 & 2775 & $87.71 \%$ & 2842 & $89.82 \%$ & 36426.47 \\
\hline \multirow[t]{4}{*}{ apex 6} & 2432 & 5000 & 2356 & $96.88 \%$ & 2374 & $97.53 \%$ & 12171.90 \\
\hline & & 10000 & 2383 & $97.99 \%$ & 2398 & $98.60 \%$ & 20083.04 \\
\hline & & 50000 & 2418 & $99.42 \%$ & 2421 & $99.55 \%$ & 91871.47 \\
\hline & & 100000 & 2424 & $99.67 \%$ & & & \\
\hline \multirow[t]{6}{*}{ apex7 } & 922 & 500 & 831 & $90.13 \%$ & 841 & $91.21 \%$ & 1086.29 \\
\hline & & 1000 & 837 & $90.78 \%$ & 848 & $91.97 \%$ & 1221.27 \\
\hline & & 5000 & 849 & $92.08 \%$ & 860 & $93.28 \%$ & 2278.55 \\
\hline & & 10000 & 859 & $93.17 \%$ & 869 & $94.25 \%$ & 3691.02 \\
\hline & & 20000 & 863 & $93.60 \%$ & 872 & $94.58 \%$ & 6785.89 \\
\hline & & ${ }^{*} 100000$ & 863 & $93.60 \%$ & 872 & $94.58 \%$ & 29931.91 \\
\hline \multirow[t]{2}{*}{ b1 } & 48 & 500 & 30 & $62.50 \%$ & 34 & $70.83 \%$ & 0.00 \\
\hline & & ${ }^{*} 100000$ & 30 & $62.50 \%$ & 34 & $70.83 \%$ & 4.01 \\
\hline \multirow[t]{3}{*}{ b9 } & 480 & 500 & 465 & $96.88 \%$ & 471 & $98.12 \%$ & 99.03 \\
\hline & & 1000 & 471 & $98.12 \%$ & 475 & $98.96 \%$ & 117.04 \\
\hline & & $* 50000$ & 471 & $98.12 \%$ & 475 & $98.96 \%$ & 1510.20 \\
\hline \multirow[t]{2}{*}{ c8 } & 930 & 1000 & 758 & $81.51 \%$ & 761 & $81.83 \%$ & 449.07 \\
\hline & & 5000 & 787 & $84.62 \%$ & 787 & $84.62 \%$ & 909.14 \\
\hline \multirow[t]{3}{*}{$\mathrm{cc}$} & 262 & 100 & 228 & $87.02 \%$ & 234 & $88.93 \%$ & 16.03 \\
\hline & & 200 & 258 & $98.47 \%$ & 259 & $98.85 \%$ & 21.02 \\
\hline & & 300 & 262 & $100.00 \%$ & 262 & $100.00 \%$ & 1.02 \\
\hline \multirow[t]{3}{*}{$\mathrm{cm} 85 \mathrm{a}$} & 176 & 100 & 156 & $88.64 \%$ & 163 & $92.61 \%$ & 7.02 \\
\hline & & 200 & 174 & $98.86 \%$ & 175 & $99.43 \%$ & 8.02 \\
\hline & & 300 & 176 & $100.00 \%$ & 176 & $100.00 \%$ & 0.00 \\
\hline \multirow[t]{4}{*}{$\mathrm{cmb}$} & 164 & 1000 & 103 & $62.80 \%$ & 106 & $64.63 \%$ & 4.01 \\
\hline & & 5000 & 138 & $84.15 \%$ & 140 & $85.37 \%$ & 9.02 \\
\hline & & 10000 & 150 & $91.46 \%$ & 150 & $91.46 \%$ & 14.02 \\
\hline & & 20000 & 152 & $92.68 \%$ & 152 & $92.68 \%$ & 25.02 \\
\hline \multirow[t]{5}{*}{ comp } & 510 & 5000 & 304 & $59.61 \%$ & 325 & $63.73 \%$ & 184.05 \\
\hline & & 10000 & 326 & $63.92 \%$ & 343 & $67.25 \%$ & 275.07 \\
\hline & & 50000 & 422 & $82.75 \%$ & 437 & $85.69 \%$ & 1055.25 \\
\hline & & 100000 & 435 & $85.29 \%$ & 450 & $88.24 \%$ & 2068.52 \\
\hline & & 200000 & 445 & $87.25 \%$ & 461 & $90.39 \%$ & 3986.19 \\
\hline cordic & 328 & 500 & 256 & $78.05 \%$ & 256 & $78.05 \%$ & 71.05 \\
\hline $\mathrm{cu}$ & 230 & 500 & 204 & $88.70 \%$ & 213 & $92.61 \%$ & 17.04 \\
\hline
\end{tabular}


TABLE III (Continued)

\begin{tabular}{cccccccc}
\hline Circuit & $\begin{array}{c}\text { \# of } \\
\text { faults }\end{array}$ & $\begin{array}{c}\text { \# of random } \\
\text { patterns }\end{array}$ & $\begin{array}{c}\text { \# of DF } \\
\text { of ckt.org }\end{array}$ & $\begin{array}{c}\text { FC of } \\
\text { ckt.org }\end{array}$ & $\begin{array}{c}\text { \# of DF } \\
\text { of ckt.rm }\end{array}$ & FC of ckt.rm & $\begin{array}{r}\text { Time } \\
(\mathrm{sec})\end{array}$ \\
\hline & 1000 & 205 & $89.13 \%$ & 214 & $93.04 \%$ & 19.02 \\
& 5000 & 211 & $91.74 \%$ & 220 & $95.65 \%$ & 37.03 \\
& 10000 & 212 & $92.17 \%$ & 221 & $96.09 \%$ & 62.03 \\
& & 212 & $92.17 \%$ & 221 & $96.09 \%$ & 264.09 \\
\hline
\end{tabular}

an index is assigned to it. By assumption, node $g_{1}$ has the largest index, so one more fanout is added to node $g_{1}$ for improving its observability. Since $g_{1} \rightarrow g_{5}$ (bold in Fig. 4(b)) can replace $g_{4} \rightarrow g_{5}$ (bold in Fig. 4(a)) whose source node $g_{4}$ has high observability, we perform this wire replacement in Figure 4(b). After rewiring, the fault simulation process is performed again, and stem line $g_{1} s-a-1$ is successfully detected by test vector $(0,1,0,1,0)$. The process will be repeated until either there is no fault remaining undetected or no further rewiring is possible.

The problem with the second approach is that, for each rewiring, the fault simulation process must be executed to ensure that the fault coverage is not devastated. It will be more efficient if there are rules to guide the wire selection and the rewiring processes such that the fault simulation process can be avoided for each rewiring. However, this is very difficult (if not impossible) since the rewiring process results in global effects to the entire circuit and no simple rules can be derived. Thus, to ensure a better solution, fault simulation needs to be performed for each rewiring process. However, we feel that this is still tolerable because the process of rewiring for testability is a one-time process and is a worthwhile effort.

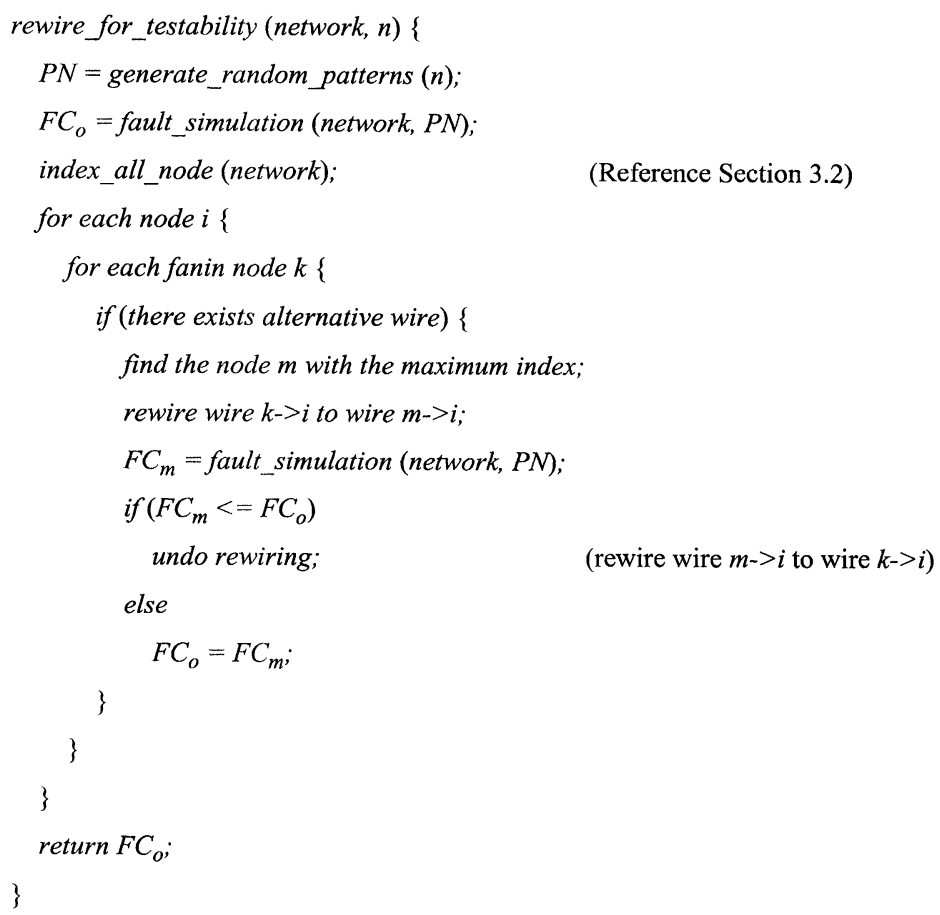

FIGURE 5 Pseudo code of approach. 


\section{SIMULATION RESULTS}

In order to evaluate the performance for the rewiring technique, the simulation software has been developed based on the SIS environment. We summarize the simulation results by Tables I, II and III. The simulation results of these three tables are generated by applying three different approaches to indexing nodes. Table I shows the simulation results by applying the first approach that assigns an index to the node of a circuit with the fanout number of each node. And Tables II and III show the simulation results by applying the second approach with the number of undetected faults on the input cone of each node indexed and the third approach with the observability of each node indexed, respectively. A pseudo-code example for approach 2 is shown in Figure 5.

Table II shows the results of computer simulation using the second approach, and it can be found that the rewiring process improves random pattern fault coverage in most cases. Note that different random test pattern lengths are used depending on the random pattern resistances of these circuits. It appears that the rewiring process is able to break the random test pattern barriers for many circuits. For example, the fault coverage of circuit ALU2 remains $84.05 \%$ after 3,000 random test patterns have been applied. In fact, we tried 50,000 random test patterns for ALU2 and failed to improve the fault coverage. However, after rewiring, the fault coverage can be easily improved to $86.21 \%$ by 1,000 random test patterns and to $87.06 \%$ by 3,000 random patterns. Each number $n$ following the asterisk $\left({ }^{*} n\right)$ in Table II indicates that the coverage can no longer be improved even by applying $n$ random test patterns. Thus, it can be observed that circuit rewiring has successfully broken the random pattern barriers for many circuits. Of course, there are cases where rewiring fails to improve the fault coverage, and other design for testability methods that really add extra circuit must be used. All experiments are done on the platform of UltaSparc workstation.

\section{CONCLUSIONS}

In this paper, we have presented a method to enhance the random pattern testability by circuit rewiring. The basic idea is to remove fanouts for gates whose observabilities have been relatively high; meanwhile, alternative wires are added to increase the observabilities for gates whose observabilities are relatively low. The wire addition and removal process does not change the circuit function. Compared with other random testability enhancement approaches, the proposed circuit rewiring technique does not introduce any hardware overhead, thus it is basically a cost-free method. Simulation results demonstrate that the rewiring technique is able to successfully break the random pattern barrier of the CUT and to improve the random test fault coverage efficiently for most circuits. The approach can also be incorporated into other random testability enhancement methods (needs hardware overhead) as a preprocess, i.e., the random testability can first be improved using the proposed method, then other methods are used to gain higher fault coverage with tolerable hardware overhead. So far, the majority of computing time is used in fault simulation for each rewiring process. The future research includes: (a) finding rules to guide circuit rewiring to avoid excessive fault simulation, or (b) using a smarter fault simulation method if the rules cannot be obtained.

\section{References}

[1] Abramovici, M., Breuer, M. A. and Friedman, A. D. (1990). Digital Systems Testing and Testable Design, Computer Science Press.

[2] Agrawal, V. D. and Seth, S. C. (1985). "Probabilistic Testability", Proc. of Int. Conf. Computer. Design: VLSI Computers, pp. $562-565$.

[3] Bardell, P. H., McAnney, W. H. and Savir, J. (1987). "Built-In Test for VLSI Pseudorandom Techniques", New York, Wiley.

[4] Chakravarty, S. and Hunt III, H. B., "On Computing Signal Probability and Detection Probability of Stuck-at Faults", IEEE Trans. on Computer, 39(11), 1369-1377, Nov., 1990.

[5] Chang, S. C., Cheng, K. T., Woo, N. S. and MarekSadowska, M., "Layout Driven Logic Synthesis for 
FPGA", Proc. Design Automation Conf., pp. 308-313, June, 1994.

[6] Chang, S. C., Marek-Sadowska, M. and Cheng, K. T., "Perturb and Simplify: Multi-Level Boolean Network Optimizer", IEEE Transaction on Computer Aided Design, 15, 1494-1504, Nov., 1996.

[7] Cheng, K. T. and Entrena, L. A., "Multi-Level Logic Optimization by Redundancy Addition and Removal", In: Proc. European Conference On Design Automation, pp. 373-377, Feb., 1993.

[8] Chakravarty, S. and Hunt III, H. B., "On Computing Signal Probability and Detection Probability of Stuck-at Faults", IEEE Trans. on Computer, 39(11), 1369-1377, Nov., 1990.

[9] Jain, S. K. and Agrawal, V. D. (1985). "Statistical Fault Analysis", IEEE Design and Test Computers, 2(2), $38-44$.

[10] Kunz, W. and Pradhan, D. K., "Multi-Level Logic Optimization by Implication Analysis", Digest Int. Conf. on Computer Aided Design, pp. 6-13, Nov., 1994.

[11] Goldstein, L. H., "Controllability/Observability Analysis of Digital Circuits", IEEE Trans. on Circuits and Systems, CAS-26, 685-693, Sep., 1979.

[12] Rohfleisch, B., Wurth, B. and Antreich, K. (1995). "Logic Clause Analysis for Delay Optimization", Proc. DAC, pp. $668-672$.

[13] Savaria, Y., Youssef, M., Kaminska, B. and Koudil, M., "Automatic Test Point Insertion for Pseudo-Random Testing", Proc. of Int. Symposium on Circuits and Systems, pp. 1960-1963, June, 1991.

[14] Schnurmann, H. D., Lindbloom, E. and Carpenter, R. F., "The Weighted Random Test-Pattern Genertator", IEEE Trans. on Computers, C-24(7), 695-700, July, 1975.

[15] Seiss, B., Trouborst, P. and Schalz, M., "Test Point Insertion for Scan-Based BIST", Proc. of European Test Conf., pp. 253-262, Apr., 1991.

[16] Tamarapalli, N. and Rajski, J., "Constructive MultiPhases Test Point Insertion for Scan-Based BIST", Proc. of Int. Test Conf., pp. 649-658, Oct., 1996.

[17] Touba, N. A. and McCluskey, E. J., "Test Point Insertion Based on Path Tracing", Proc. of VLSI Test Symposium, pp. 2-8, Apr., 1996.

[18] Tsai, H. C., Cheng, K. T., Lin, C. J. and Bhawmik, S. (1997). "A Hybrid Algorithm for Test Point Selection for Scan-Based BIST", Design Automation Conf., pp. $478-483$.

[19] Wunderlich, H. J., "Self Test Using Unequiprobable Random Patterns", Digest of Papers 17th Intn. Symp. on Fault-Tolerant Computing, pp. 258-263, July, 1987.

\section{Authors' Biographies}

Sih-Chieh Chang received the B.S. degree in Electrical Engineering from National Taiwan University in 1987 and the Ph.D. degree in Electrical Engineering from the University of California, Santa Barbara, in 1994. He worked at Synopsys, Inc., Mountain View, Ca, from 1995 to 1996. He then joined the faculty at the Institute of
Computer Science and Information Engineering, National Chung, Cheng University, Taiwan. His current research interests include VLSI logic synthesis, layout, and FPGA related applications. Dr. Chang received a Best Paper Award at the 1994 Design Automation Conference.

Kwen-Yo Chen was born in Taiwan, Republic of China, He received the B.S.C.S degree from National Ching Hwa University, Taiwan, in 1996. Currently, he is serving in the Army, Taiwan, Republic of China. His research interesting include VLSI circuit and microprocessor testing.

Ching-Hwa Cheng was born in Taiwan, Republic of China, $\mathrm{He}$ received the M.S.E.E degree from Chung Hwa University, Taiwan, in 1993. He received the Ph.D. degree in the Department of Computer Science and Information Engineering, National Chung Cheng University, Chiayi, Taiwan in 2000. His research interesting include VLSI circuit testing and fault tolerant computing.

Wen-Ben Jone was born in Taiwan, Republic of China. He received the $\mathrm{Ph} . \mathrm{D}$. degree in computer engineering and science from Case Western Reserve University, in 1987. He is currently with the Department of Electrical and Computer Engineering and Computer Science, University of Cincinnati, Cincinnati, OH 45221, USA. His research interests include fault-tolerant computing, VLSI design and test. He has published more than 85 papers and served as a review in these research areas in various technical journals and conferences. Dr. Jone received the Best Thesis Award from The Chinese Institute of Electrical Engineering (Republic of China), in 1981.

Sunil R. Das is a professor of Electrical and Computer Engineering at the School of Information Technology and Engineering, University of Ottawa, Ottawa, Ontario, Canada. He holds a B.Sc.(Honors) in Physics and an M.Sc.(Tech) and a Ph.D. in Radiophysics and Electronics from the University of Calcutta, Calcutta, West Bengal, India. He previously held academic and research position with the Department of Electrical Engineering and Computer Science, Computer 
Science Division, University of California, Berkeley, CA, the Centre for Reliable Computing $(C R C)$, Computer Systems Laboratory, Department of Electrical Engineering Stanford University, Stanford, CA (on Sabbatical leave), the Institute of Computer Engineering, National Chiao Tung University, Hsinchu, Taiwan, Republic of China, and the Center of Adavnced Study $(C A S)$, Institute of Radiophysics and Electronics, University of Calcutta.

Dr. Das published extensibly in the areas of switching and automata theory, digital logic design, threshold logic, fault-tolerant computing, microprogramming and microarchitecture, microcode optimization, applied theory of graphs, and combinatorics. He served in the Technical Program Committees and Organizing Committees of many IEEE and non-IEEE International Conferences, Symposia, and workshops, and also acted as session organizer, session chair, and panelist. Dr. Das was elected one of the delegates of the prestigious GOOD PEOPLE, GOOD DEEDS of the Republic of China in 1981 in recognition of his outstanding contributions in the field of research and education. $\mathrm{He}$ is listed in the MARQUIS WHO'S WHO Biographical Directory of the Computer Graphics Industry, Chicago, IL (First Edition, 1984).

Dr. Das served as the Managing Editor of the IEEE VLSI Technical Bulletin, a Publication of the IEEE Computer Society Technical Committee (TC) on VLSI, and an Executive Committee Member of the IEEE Computer Society Technical Committee (TC) on VLSI. Dr. Das is currently an Associate Editor of the IEEE Transactions on Systems, Man, and Cybernetics, an Associate Editor of the IEEE Transactions on VLSI Systems, and a Member of the Editorial Board and a Regional Editor for Canada of the VLSI Design: An International Journal of Custom-Chip Design, Simulation and Testing published by Gordon and Breach Science Publishers, Inc., Ny. Dr. Das is a former Administrative Committee (ADCOM) Member of the IEEE Systems, Man, and Cybernetics Society, a former Associate
Editor of the SIGDA Newsletter, the publication of the ACM Special Interest Group on Design Automation, and a former Associate Editor of the International Journal of Computer Aided VLSI Design published by Ablex Publishing Corporation, Norwood, NJ. He was the Associate Guest Editor of the IEEE Journal of Solid-State Circuits Special Issues on Microelectronic Systems (Third and Fourth Special Issues), and Guest Editor of the International Journal of Computer Aided VLSI Design (September, 1991) as well as VLSI Design: An International Journal of Custom-Chip Design, Simulation and Testing (March, 1993 and September, 1996) Special Issues on VLSI Testing. Dr. Das edited jointly with P. K. Srimani a book entitled, Distributed Mutual Exclusion Algorithms, published by the IEEE Computer Society Press. Los Alamitos, CA 1992 in its Technology Series. He is also the author jointly with C. L. Sheng of a text on Digital Logic Design being published by Ablex Publishing Corporation. Dr. Das serves as the Co-Chair of the IEEE Computer Society Students Activities Committee from Region 7 (Canada).

Dr. Das is a Fellow of the Institute of Electrical and Electronics Engineers (IEEE), Inc. (with Membership in the IEEE Computer Society, IEEE Systems, Man, and Cybernetics Society, IEEE Circuits and Systems Society, and IEEE Instrumentation and Measurements Society, and a Member. of the Association for Computing Machinery ( $A C M)$, U.S.A. He was elected a Fellow of the IEEE in 1994 for Contributions to switching theory and computer design.

Dr. Das is the 1996 recipient of the IEEE Computer Society's highly esteemed Technical Achievement Award for his Pioneering Contributions in the fields of switching theory and modern digital design, digital circuits testing, microarchitecture and microprogram optimization, and combinatorics and graph theory. $\mathrm{He}$ is also the 1997 recipient of the IEEE Computer Society's Meritorious Service Award for excellent service contribution to Transactions on VLSI System and the Society, and was elected a Fellow of the Society for Design and Process Science, U.S.A. in 1998 for his 
accomplishments in integration of disciplines, theories and methodologies, development of scientific principles and methods for design and process science as applied to traditional disciplines of engineering, industrial leadership and innovation, and educational leadership and creativity.

Dr. Das presently serves as a Member of the IEEE Computer Society Fellow Evaluation Committee. 

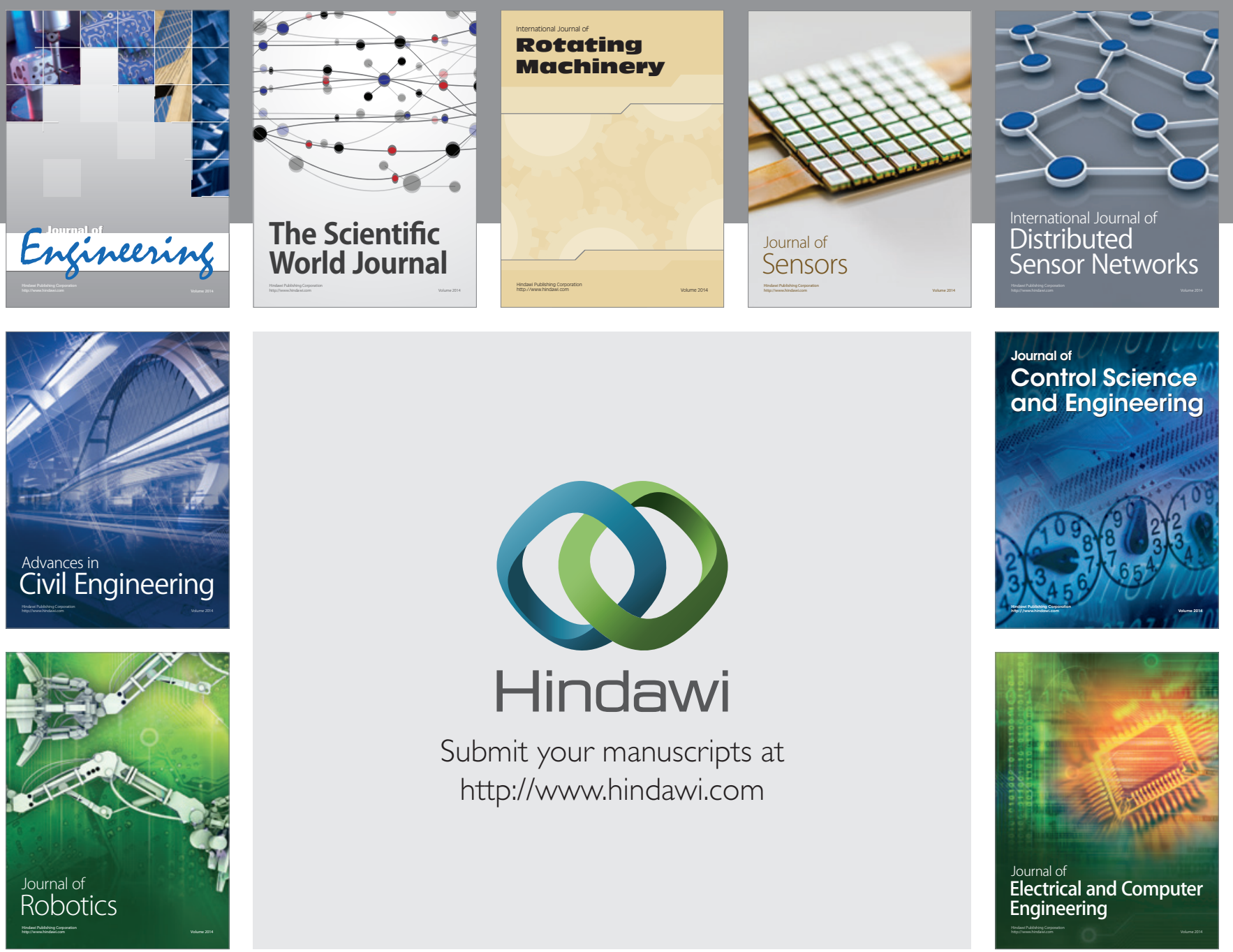

Submit your manuscripts at

http://www.hindawi.com
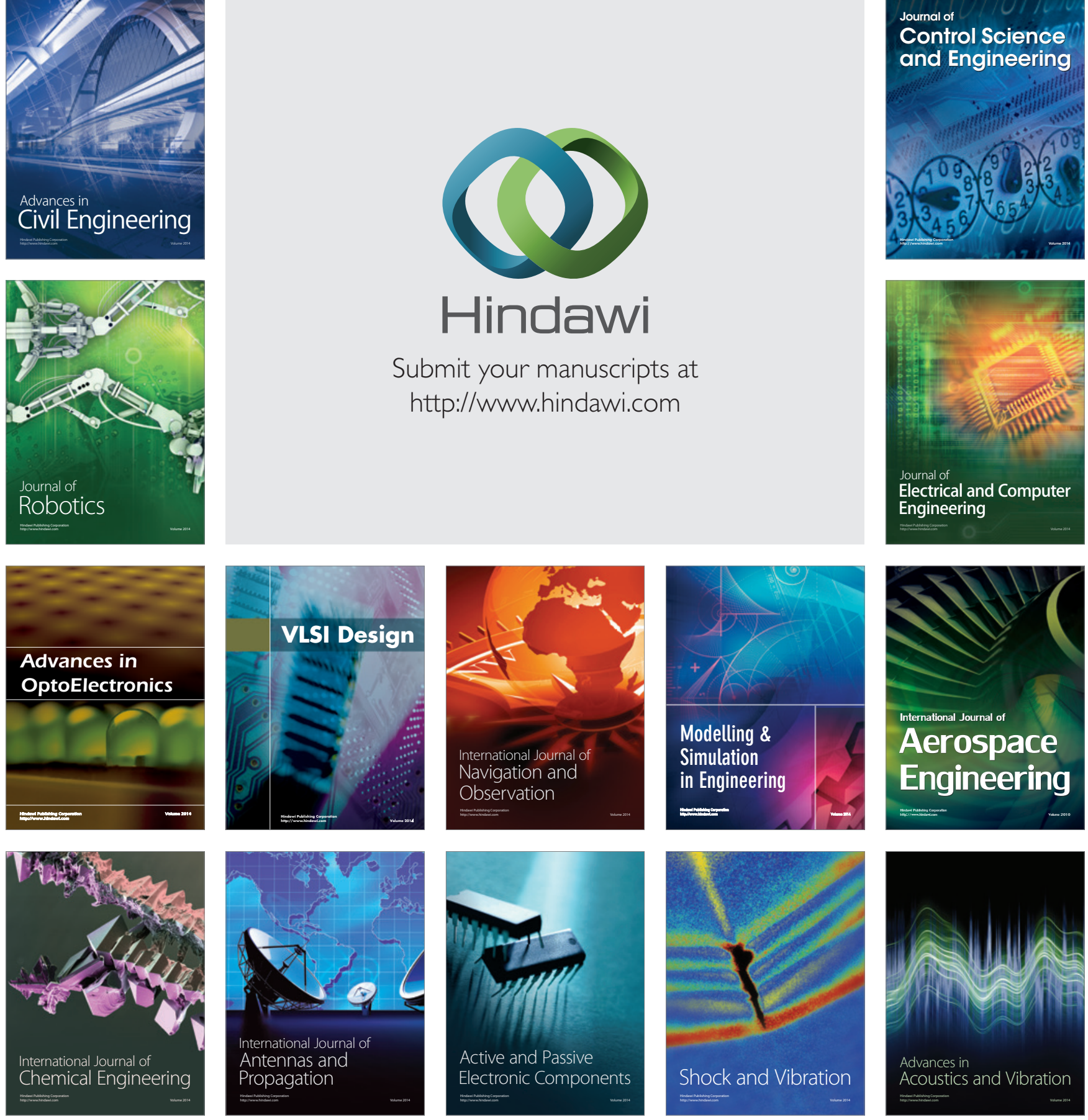